\title{
CERT depletion predicts chemotherapy benefit and mediates cytotoxic and polyploid specific cancer cell death through autophagy induction
}

Lee, Alvin J. X.; Roylance, Rebecca; Sander, Jil; Gorman, Patricia; Endesfelder, David; Kschischo, Maik; Jones, Neil P.; East, Philip; Nicke, Barbara; Spassieva, Stefka

Total number of authors:

20

Published in:

Journal of Pathology

Link to article, DOI:

10.1002/path.2998

Publication date:

2012

Document Version

Publisher's PDF, also known as Version of record

Link back to DTU Orbit

Citation $(A P A)$ :

Lee, A. J. X., Roylance, R., Sander, J., Gorman, P., Endesfelder, D., Kschischo, M., Jones, N. P., East, P. Nicke, B., Spassieva, S., Öbeid, L. M., Birkbak, N. J., Szallasi, Z., McKnight, N. C., Rowan, A. J., Speirs, V., Hanby, A. M., Downward, J., Tooze, S. A., \& Swanton, C. (2012). CERT depletion predicts chemotherapy benefit and mediates cytotoxic and polyploidspecific cancer cell death through autophagy induction. Journal of Pathology, 226(3), 482-494. https://doi.org/10.1002/path.2998

\section{General rights}

Copyright and moral rights for the publications made accessible in the public portal are retained by the authors and/or other copyright owners and it is a condition of accessing publications that users recognise and abide by the legal requirements associated with these rights.

- Users may download and print one copy of any publication from the public portal for the purpose of private study or research.

- You may not further distribute the material or use it for any profit-making activity or commercial gain

- You may freely distribute the URL identifying the publication in the public portal 


\title{
CERT depletion predicts chemotherapy benefit and mediates cytotoxic and polyploid-specific cancer cell death through autophagy induction
}

\author{
Alvin J. X. Lee,' Rebecca Roylance, ${ }^{2}$ Jil Sander, 1,3 Patricia Gorman, ${ }^{2}$ David Endesfelder, 1,3 Maik Kschischo,3 \\ Neil P. Jones, ${ }^{4}$ Philip East,' Barbara Nicke,' Stefka Spassieva, ${ }^{5}$ Lina M. Obeid,,6 Nicolai Juul Birkbak, ${ }^{7,8}$ \\ Zoltan Szallasi,79 Nicole C. McKnight,' Andrew J. Rowan,' Valerie Speirs, ${ }^{10}$ Andrew M. Hanby'0 \\ Julian Downward,' Sharon A. Tooze' and Charles Swanton!,II* \\ Cancer Research UK London Research Institute, 44 Lincoln's Inn Fields, London WC2A 3LY, UK \\ 2 Barts Cancer Institute, Queen Mary University of London, Charterhouse Square, London ECIM 6BQ, UK \\ 3 University of Applied Sciences Koblenz, RheinAhrCampus, Südallee 2, 53424 Remagen, Germany \\ ${ }^{4}$ Cancer Research Technology, Wolfson Institute for Biomedical Research, University College London, London WCIE 6BT, UK \\ ${ }^{5}$ Department of Medicine, Medical University of South Carolina, Charleston, SC 29425, USA \\ ${ }^{6}$ Division of General Internal Medicine, Ralph H. Johnson Veterans Affairs Hospital, Charleston, SC 2940 I, USA \\ ${ }^{7}$ Centre for Biological Sequence Analysis, Technical University of Denmark, DK-2800 Lyngby, Denmark \\ ${ }^{8}$ Department of Cancer Biology, Dana-Farber Cancer Institute, Harvard Medical School, Boston, MA 02 I I 5, USA \\ 9 Children's Hospital Informatics Program at the Harvard-MIT Division of Health Sciences and Technology, Harvard Medical School, Boston, MA \\ 02115 , USA \\ 10 Leeds Institute of Molecular Medicine, University of Leeds, Leeds LS9 7TF, UK \\ "I UCL Cancer Institute and Macmillan Cancer Centre, Huntley Street, London WCIE 6BT, UK
}

*Correspondence to: Charles Swanton, Cancer Research UK London Research Institute, 44 Lincoln's Inn Fields, London WC2A 3LY, UK. e-mail: charles.swanton@cancer.org.uk

\begin{abstract}
Chromosomal instability (CIN) has been implicated in multidrug resistance and the silencing of the ceramide transporter, CERT, promotes sensitization to diverse cytotoxics. An improved understanding of mechanisms governing multidrug sensitization might provide insight into pathways contributing to the death of CIN cancer cells. Using an integrative functional genomics approach, we find that CERT-specific multidrug sensitization is associated with enhanced autophagosome-lysosome flux, resulting from the expression of LAMP2 following CERT silencing in colorectal and HER2 ${ }^{+}$breast cancer cell lines. Live cell microscopy analysis revealed that CERT depletion induces LAMP2-dependent death of polyploid cells following exit from mitosis in the presence of paclitaxel. We find that CERT is relatively over-expressed in HER2 ${ }^{+}$breast cancer and CERT protein expression acts as an independent prognostic variable and predictor of outcome in adjuvant chemotherapy-treated patients with primary breast cancer. These data suggest that the induction of LAMP2-dependent autophagic flux through CERT targeting may provide a rational approach to enhance multidrug sensitization and potentiate the death of polyploid cells following paclitaxel exposure to limit the acquisition of CIN and intra-tumour heterogeneity. Copyright $\odot 201$ I Pathological Society of Great Britain and Ireland. Published by John Wiley \& Sons, Ltd.
\end{abstract}

Keywords: CERT; autophagy; LAMP2; HER2; polyploidy; drug resistance

Received 24 July 20 I I; Revised 5 September 20 I ; Accepted 8 September 20 I I

No conflicts of interest were declared.

\section{Introduction}

Drug resistance is associated with significant treatment morbidity and impairment of quality of life. The ceramide pathway has been implicated in the cellular response to cytotoxic chemotherapy, and enzymes which impair ceramide accumulation in response to drug exposure have been associated with multidrug resistance [1]. We recently identified the ceramide transporter CERT through a multidrug sensitivity RNAi screen that enhanced sensitivity to paclitaxel, cisplatin, 5-FU and doxorubicin when silenced from multiple cancer cell lines [HCT116 (colon carcinoma), MDA-MB-231 (breast adenocarcinoma) and A549 (non-small cell lung carcinoma)] [2]. CERT transports ceramide from the endoplasmic reticulum (ER) to the Golgi apparatus where it is converted into sphingomyelin [3]. We found that CERT was relatively over-expressed in paclitaxel-resistant ovarian cancer and was one of six genes included in a functional metagene, predictive of combination-chemotherapy drug sensitivity in breast cancer [4].

Through the same RNAi screen, we identified several genes whose depletion induced both polyploidy 
and paclitaxel resistance, indicating that pathways monitoring chromosomal stability and taxane sensitivity may be related. Consistent with this hypothesis, we have confirmed that CIN is associated with paclitaxel resistance in a small clinical trial cohort of patients with ovarian cancer [5]. Follow-up analysis has revealed that CIN appears to be associated with multidrug resistance that we hypothesize may result from tumour heterogeneity between cancer cells fostering diversity and evolutionary robustness or an intrinsic aneuploidy tolerant state [6].

We reasoned that understanding the molecular pathways responsible for cell death following CERT depletion and paclitaxel exposure may offer a broader insight into mechanisms regulating mitotic catastrophe and suggest therapeutic opportunities to specifically limit the development of chromosomally unstable cells. Here, through parallel integrative genomics approaches, we investigate the role of CERT in cancer drug-induced cell death.

\section{Materials and methods}

\section{siRNA transfections and paclitaxel treatment}

All siRNAs were obtained from Dharmacon. Unless otherwise stated, all siRNA transfections were performed using reverse transfection methods with Lipofectamine RNAiMax reagent (Invitrogen) at $25 \mathrm{nM}$ final concentration of siRNA, and after $48 \mathrm{~h}$ of siRNA transfection the cells were treated with $25 \mathrm{nM}$ paclitaxel or vehicle control.

\section{CERT microarray expression profiling}

HCT116 cells were transfected with either siCON or siCERT and treated in triplicate with paclitaxel, C6-ceramide or vehicle control for $8 \mathrm{~h}$ for use on HuEx-1_0-st-v2 exon arrays (Affymetrix) (for detailed analysis of exon array, see Supporting information, Supplementary methods).

Taqman Gene Expression Assays (Applied Biosystems) were used according to the manufacturer's instructions in order to validate gene expression using qPCR.

\section{Cell line drug response assays}

Cells were plated into 96-well tissue culture microplates at an initial seeding density of 4000 cells/well for siRNA reverse transfection. After $48 \mathrm{~h}$ of paclitaxel or vehicle control treatment, cell viability was assayed using the Celltiter-Blue Cell Viability Assay (Promega), according to the manufacturer's instructions.

\section{LC3 immunoblotting}

Cells were plated into six-well tissue culture microplates at an initial seeding density of 300000 cells/well for siRNA reverse transfection. The cells were then harvested at $24 \mathrm{~h}$ after paclitaxel or vehicle control treatment or at other specified time points. Immunoblotting was performed with primary anti-LC3 antibodies (Nanotools), using standard immunoblotting protocols. Where relevant, the protease inhibitor leupeptin (Sigma-Aldrich) was used at a final concentration of $1 \mu \mathrm{g} / \mathrm{ml}$.

\section{Immunofluorescence microscopy}

Cells were fixed using PTEMF (20 mM PIPES, pH 6.8, $10 \mathrm{mM}$ EGTA, $1 \mathrm{mM} \mathrm{MgCl}_{2}, 0.2 \%$ Triton X100 and $4 \%$ formaldehyde) for $10 \mathrm{~min}$ at room temperature. Primary antibodies were diluted as follows in PBS + 3\% BSA anti-LAMP2 (Abcam) 1/1000. Crossadsorbed secondary antibodies were used (Molecular Probes). 3D image stacks were acquired in $0.2 \mu \mathrm{m}$ steps, using a $\times 100$ oil NA 1.4 objective on an Olympus Deltavision Personal microscope (API) equipped with a DAPI-FITC-Rhod/TR-CY5 filter set (Chroma) and a Coolsnap HQ camera. The 3D image stacks were deconvolved with SoftWorx (API).

\section{Live cell imaging}

For live cell imaging H2B-mRFP HCT116 cells were monitored at $37^{\circ} \mathrm{C}$ and $5 \% \quad \mathrm{CO}_{2}$ in LabTech II (LabTech) chambers. Cells were plated into chamber slides for reverse transfection and treated with paclitaxel or vehicle control. Images were acquired every $10 \mathrm{~min}$ for $48 \mathrm{~h}$ using a $\times 40$ oil NA 1.3 objective on an Olympus Deltavision Personal microscope (API) equipped with a DAPI-FITC-Rhod/TR-CY5 (Chroma) filter set and environmental chamber.

\section{Tissue microarray cohort}

Archival paraffin-embedded breast cancer tissue was obtained from 356 patients diagnosed at Leeds Teaching Hospitals NHS Trust between 1983 and 1997. Tissue microarrays (TMAs) were constructed containing cores, $0.6 \mathrm{~mm}$ in diameter and $4 \mu \mathrm{m}$ in thickness, selected from representative tumour areas as determined by a consultant breast histopathologist $(\mathrm{AMH})$ from haematoxylin and eosin (H\&E)-stained sections. Full clinico-pathological data was available. The majority of chemotherapy-treated patients were given cyclophosphamide, methotrexate and fluorouracil 5-FU (CMF) or fluorouracil 5-FU, epirubicin and cyclophosphamide (FEC) treatment. Ethical approval was obtained (Leeds East 06/Q1206/180).

Following deparaffinization, an antigen retrieval step was performed. Tissue sections were incubated with CERT rabbit polyclonal antibody (Bethyl Laboratories), dilution 1:3500 for $1 \mathrm{~h}$. Biotinylated secondary antibodies were used followed by streptavidin-biotin-peroxidase complex. Peroxidase was localized using diaminobenzidine, with counterstaining with Mayer's haematoxyin. A known positive control was used with each batch of staining. Slides were 
viewed by two independent scorers blinded to each other's findings.

CERT localized to the cytoplasm. Scoring was performed for both the percentage and intensity of staining, using a scale of $0-3$ (with $0=$ tumours with no staining whatsoever; $1=$ tumours with rare scattered positive staining; $2=$ tumours with moderate to strong staining but with some cells showing no readily detected staining; and $3=$ tumours with strong staining, uniformly visualized over the whole section). A score of percentage positive staining cells $\times$ the intensity score was calculated and this was defined as the quickscore [7].

A maximally selected rank statistic [8] was used on all 356 patients to detect a CERT expression quickscore threshold for separating good from poor prognosis. The R-package maxstat [9] was used to derive the reported threshold of 175 . The reported $p$ value of 0.0030 (Figure 5A) is conservative and adjusted for the selection of the threshold, and thus avoids the problem of reporting over-optimistic low $p$ values arising from the selection process. Based on this threshold (quickscore $=175$ ), CERT expression was grouped into high and low expression for the whole dataset. Survival analysis was performed with a univariate Cox-proportional hazard regression and a log-rank statistic was used for significance testing between the CERT-high and CERT-low groups.

\section{Results}

\section{CERT depletion results in ceramide accumulation}

CERT silencing was previously reported to sensitize the HCT116 colorectal cancer, MDA-MB-231 breast and A549 non-small cell lung cancer cell lines to multiple clinically relevant cytotoxics in a kinome library RNAi screen [2]. CERT transports longchain ceramides $\left(\mathrm{C}_{14-20}\right)$ and short-chain fluorescent ceramide analogues from the endoplasmic reticulum to the Golgi apparatus [3]. We hypothesized that CERT silencing in the presence of paclitaxel might mimic the effect of ceramide exposure through the attenuation of ceramide transport from the endoplasmic reticulum (ER) to the Golgi and its subsequent conversion into sphingomyelin. We confirmed that depletion of CERT by RNAi ( uation of ceramide transport with ceramide confined to membranous and diffuse cytoplasmic structures, in contrast to control transfected cells ( $\mathrm{siCON}$ ), in which ceramide is localized to Golgi-like punctate perinuclear regions (see Supporting information, Figure S1A) [10].

To establish whether CERT depletion might induce alterations in ceramide levels that could be exacerbated by paclitaxel exposure, we analysed lipid extracts for ceramide content by mass spectrometry. Paclitaxel treatment ( $25 \mathrm{nM}$ for $48 \mathrm{~h}$ ) of siCON-transfected cells did not significantly increase total ceramide levels (see Supporting information, Figure S1B). CERT depletion resulted in an increase in total ceramide levels relative to siCON-transfected cells ( $p=0.008$ ) and the combination of siCERT with paclitaxel treatment resulted in a synergistic increase in total cellular ceramide levels ( $p=0.0001)$ relative to CERT silencing or paclitaxel treatment alone.

\section{Transcriptomic analysis reveals a CERT-specific response}

We hypothesized that the CERT-specific paclitaxel sensitization phenotype may be attributable to two mechanisms; the first primes the cell for paclitaxelinduced cell death following CERT silencing, the second mechanism might be attributable to a response derived from ceramide sequestration within the ER that acts synergistically to enhance cell death. We reasoned that through the identification of a CERT-specific and ER-restricted ceramide transcriptional response, mediators of this process might be identifiable.

First, we addressed whether CERT depletion is associated with a specific transcriptional response that might inform upon the mechanism through which CERT silencing primes cells for paclitaxel-induced death. We used microarray expression analysis of HCT116 cells to identify genes differentially regulated following CERT depletion in the presence of ceramide, paclitaxel or DMSO vehicle control compared to the same treatments in siCON-transfected cells in three independent experiments. We identified a common CERT-specific transcriptional response (Figure 1A) and 24 genes from this analysis were selected for qPCR validation. The analysis was performed on the same triplicate biological samples used for the microarray analysis, and we found that microarray profiles correlated closely with gene expression changes validated by qPCR $(R=0.97)$, as previously described [11], indicating the reliability of gene expression changes quantified by Affymetrix HuEx-1_0-st-v2 array profiling (Figure 1B).

\section{CERT depletion induces LAMP2, which is required for paclitaxel cell death}

Genes expressed following CERT depletion might prime cells for subsequent cell death initiated by paclitaxel. Silencing the expression of these genes through RNAi might antagonize paclitaxel response and siCERT-specific paclitaxel sensitization. Seven genes (CPA4,FYCO1,KLF6,LAMP2,MDK,WDFY1 and WIPI1), whose expression was induced following CERT depletion (and validated by qPCR), were silenced by RNAi in HCT116 cells and paclitaxel sensitivity was assessed. Two of the seven genes promoted paclitaxel resistance when silenced in HCT116 cells: $L A M P 2$, encoding a lysosomal membrane protein [12]; and CPA4, a carboxypeptidase with unknown specific substrate (Figure 1C). CERT silencing induced a $0.56 \log _{2}$-fold change in levels of LAMP2 expression (Figure 1B), corresponding to a $47 \%$ increase in the expression of LAMP2. The induction of LAMP2 
(A)

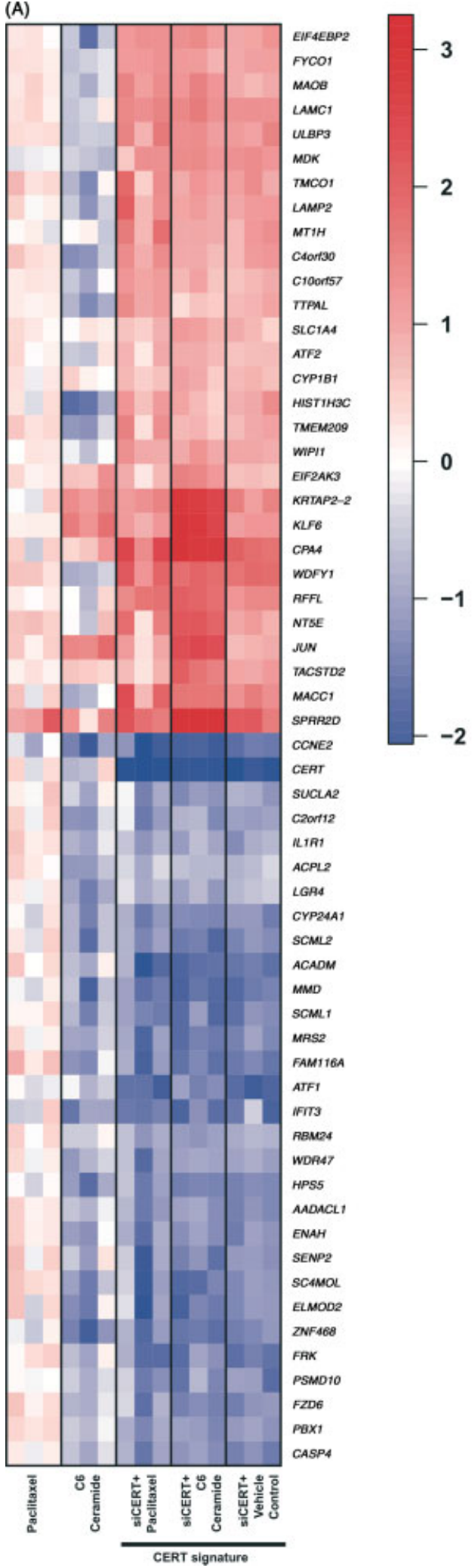

(B)

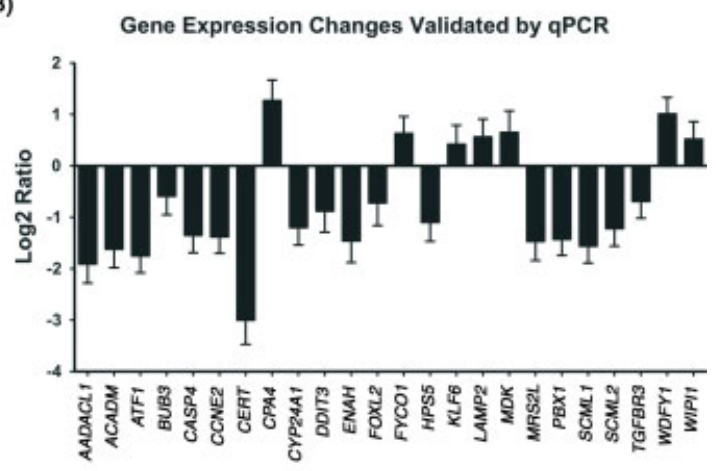

(C)

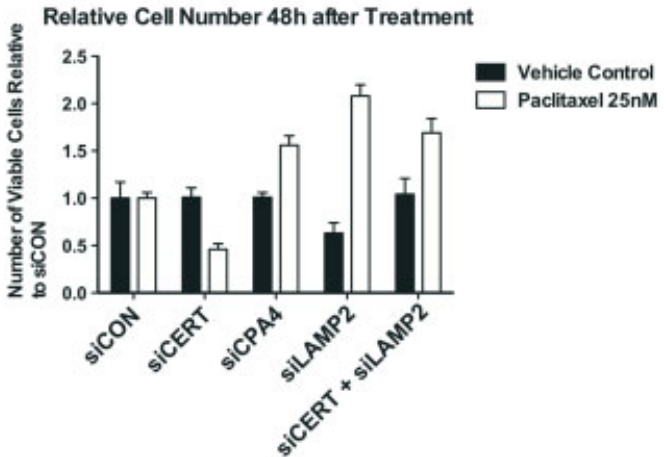

(D)
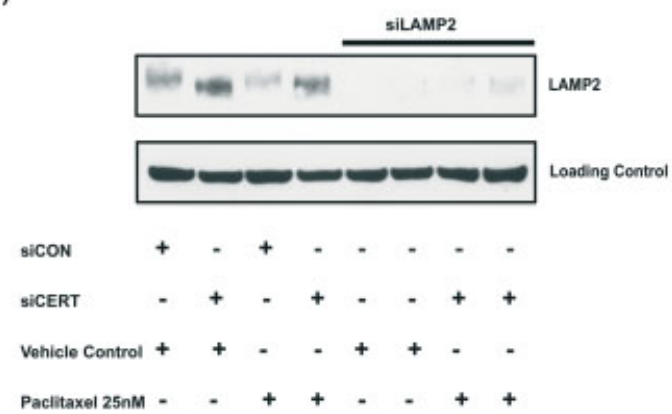

(E)

Gene Expression Changes Relative to siCON

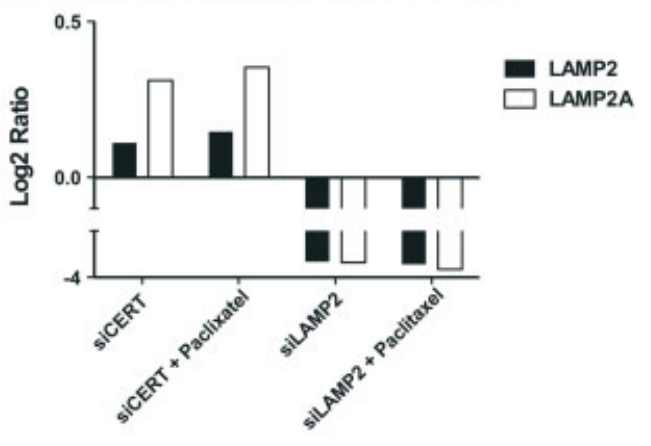

Figure 1. CERT gene expression signature and investigation of pathways mediating paclitaxel-induced cell death. (A) Heat map of differentially expressed genes in HCT116 cells $8 \mathrm{~h}$ after paclitaxel, C6-ceramide or vehicle control treatment, $48 \mathrm{~h}$ following siCERT transfection. Expression of this gene signature in siCON-transfected paclitaxel and C6-ceramide-treated cells provided for comparison. (B) Graph of qPCR validation of 24 genes that are differentially expressed following CERT depletion. Error bars represent standard deviation (SD) across triplicate experiments. (C) Graph of relative cell number following transfection of siCON, siCERT, siCPA4, siLAMP2 or siCERT and siLAMP2 and vehicle control or paclitaxel treatment. Data were normalized to cell number in siCON/vehicle control or siCON/paclitaxel for paclitaxel-treated experiments. Error bars represent SD across six replicates/condition. (D) Western blot analysis showing LAMP2 protein expression $24 \mathrm{~h}$ after paclitaxel or vehicle control treatment following $48 \mathrm{~h}$ of siCON or siCERT transfection. (E) Graph of qPCR assessment of gene expression of LAMP2 and LAMP2A specifically. 
protein following CERT silencing and efficient knockdown of LAMP2 protein by RNAi was confirmed (Figure 1D). qPCR quantification using primers specific to the LAMP2A isoform and to all LAMP2 splice variants revealed an increase in expression of LAMP2, with a greater specific increase in the LAMP2A isoform (Figure 1E). To assess whether siCERT-mediated paclitaxel sensitization was dependent upon LAMP2 expression, both CERT and LAMP2 were co-depleted and paclitaxel sensitivity was assessed. siLAMP2 abrogated the ability of siCERT to sensitize cells to paclitaxel exposure (Figure 1C). Co-silencing of both CERT and LAMP2 was confirmed by qPCR (see Supporting information, Figure S1C), and antagonizm of paclitaxel was confirmed for two separate siLAMP2 siRNA oligonucleotide sequences (see Supporting information, Figure S1D).

\section{Ceramide treatment following CERT silencing initiates a specific transcriptional response}

CERT depletion attenuates the transport of exogenous ceramide from the ER to the Golgi and promotes a synergistic increase in cellular ceramide levels with paclitaxel. We hypothesized that the rise in cellular ceramide, restricted to the ER compartment following CERT depletion, might contribute to cellular cytotoxicity coordinated with a CERT-specific transcriptional response.

To investigate the transcriptional response attributable to ceramide that is likely to be restricted to the ER following CERT silencing, we asked what gene sets were significantly altered in HCT116 cells treated with ceramide on a CERT-depleted background, relative to ceramide-treated siCON-transfected cells. The autophagy process network (Metacore, GeneGo Inc.) was significantly altered following ceramide treatment of CERT-depleted cells compared to ceramide treatment of HCT116 cells with proficient CERT transport (Figure 2A; $p=3.5 \mathrm{E}-05$ ).

\section{CERT depletion and paclitaxel exposure initiates an autophagy response}

Given the observations that: (a) CERT depletion induces a synergistic rise in cellular ceramide in the presence of paclitaxel; (b) ceramide treatment of CERT-depleted cells results in an autophagic transcriptional response; and (c) LAMP2, induced following CERT depletion, can antagonize paclitaxel cell death when silenced from cells, we addressed whether autophagy induction might occur following paclitaxel treatment of CERT-depleted cells. Lipidation of endogenous microtubule-associated protein light chain 3 (LC3) was studied in HCT116 cells following CERT silencing and paclitaxel exposure. Following transfection of siCON and paclitaxel treatment, there was minimal change in LC3-I (unlipidated LC3) or LC3-II (lipidated LC3) levels compared to vehicle control-treated HCT116 cells (Figure 2B). Similarly, CERT silencing in the absence of drug treatment did not significantly alter LC3-I or LC3-II levels. The combination of CERT silencing and paclitaxel treatment resulted in a reduction of both LC3-I and LC3-II, suggestive of autophagy flux induction. Consistent with the degradation of LC3 by lysosomal proteases, addition of the lysosomal protease inhibitor, leupeptin, resulted in the reappearance of LC3-I and LC3-II in siCERT cells treated with paclitaxel (see Supporting information, Figure S1E).

\section{CERT-mediated paclitaxel sensitization is LAMP2-dependent}

It has been proposed that the tethering of the autophagosome to the lysosomal membrane is a ratelimiting step that defines the life-span of autophagosomes [13]. The expression of LAMP2, encoding a lysosomal membrane protein [12], is induced following CERT depletion and LAMP2 silencing antagonizes paclitaxel sensitization on a CERT depleted background. Given the role of lysosomal proteases in taxane sensitivity $[14,15]$, we speculated that LAMP2 may contribute to paclitaxel sensitization through autophagic cell death. Supporting the role of LAMP2 in siCERT/paclitaxel-induced autophagy response and LC3 degradation, LAMP2 silencing attenuated LC3 degradation following CERT and LAMP2 co-depletion (Figure 2B).

Cells were assayed at 12, 24, 48 and $72 \mathrm{~h}$ for apoptotic activity, as measured by caspase $3 / 7$ activity, and for LC3-I and LC3-II expression (Figure 2C). Maximal reduction of LC3-I and LC3-II following siCERT and paclitaxel was observed at $12 \mathrm{~h}$ with reappearance of both moieties by $48 \mathrm{~h}$. In contrast, maximal apoptotic activity of siCERT- and siCON-transfected cells was observed $36 \mathrm{~h}$ later, at $48 \mathrm{~h}$ following paclitaxel treatment, suggesting that autophagic flux precedes caspase activity following paclitaxel exposure of CERTdepleted cells.

CERT depletion induces localization of LAMP2/LC3 and the accumulation of lysosomal structures in the presence of paclitaxel

The localization of endogenous LAMP2 expression was addressed following CERT depletion in GFPLC3-HEK293 cells, which stably express GFP-LC3, by fixed cell immunofluorescence in the presence of the lysosomal acidification inhibitor Bafilomycin A1 to prevent GFP-LC3 degradation. We observed elevated endogenous LAMP2 intensity, consistent with an increase in LAMP2 expression, and co-localization of GFP-LC3 with LAMP2 in siCERT/paclitaxeltreated relative to siCON/paclitaxel HEK293 cells (Figure 2D). Depletion of CERT resulted in a significant increase in LAMP2 spot intensity (Mann-Whitney U-test, $p=0.015)$, suggesting that lysosomal size increases following CERT depletion and paclitaxel treatment.

Electron microscopy (EM) analysis revealed an increase in the number of cells with electron-dense structures that may correspond to lysosomal or 

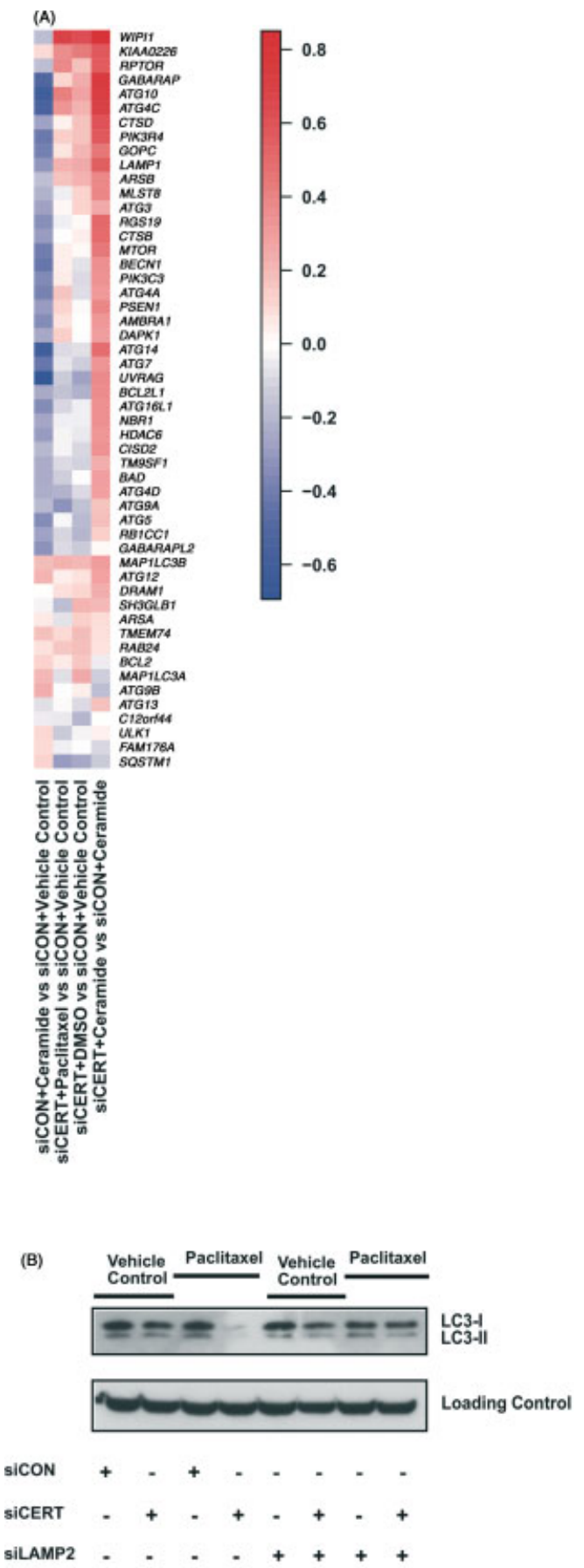

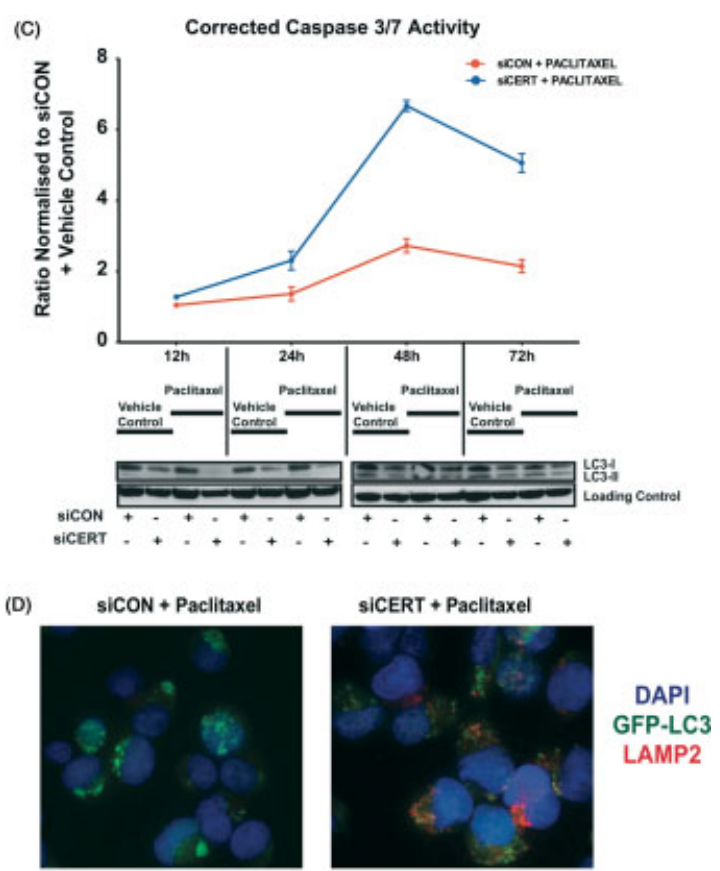

(E)

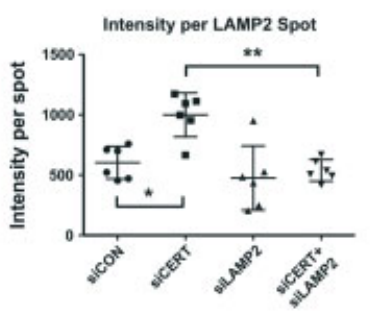

(F)

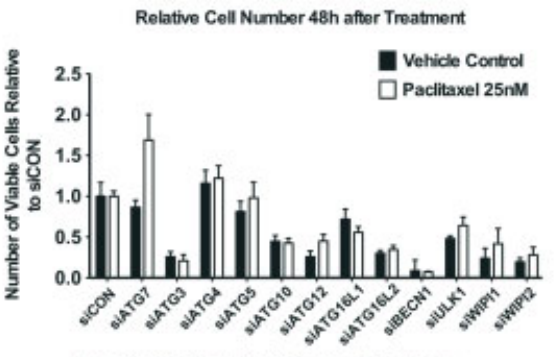

(G)

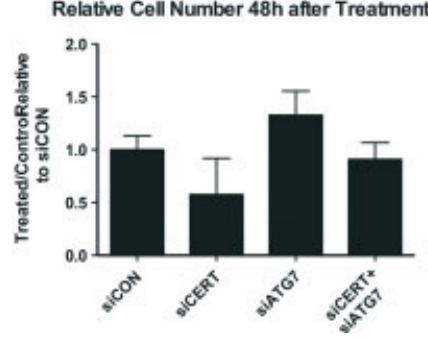

Figure 2. siCERT/paclitaxel initiates an autophagy-like response. (A) Heat map of autophagy gene set (as defined using Metacore from GeneGo Inc.). Autophagy gene set was found to be differentially regulated in CERT-depleted cells treated with ceramide compared to ceramide treatment of HCT116 cells with proficient CERT transport. (B) LC3I/II western blot analysis following CERT and LAMP2 silencing and $24 \mathrm{~h}$ of paclitaxel exposure. (C) Graph showing corrected Caspase 3/7 activity of siCON/paclitaxel- and siCERT/paclitaxel-treated HCT116 cells normalized to siCON/vehicle control over $72 \mathrm{~h}$. Error bars represent SD over six replicates/condition. Below the graph is a western blot analysis over the same time course, showing LC3 expression following siCON or siCERT transfection with vehicle control or paclitaxel treatment. (D) Representative immunofluorescence microscopy images of siCON/paclitaxel and siCERT/paclitaxel cells in the presence of Bafilomycin A1. Blue corresponds to DAPI staining, green to GFP-LC3 and red to LAMP2. (E) Scatter plot of LAMP2 intensity per spot following siCON, siCERT, siLAMP2 or siCERT and siLAMP2 silencing and treatment with $25 \mathrm{nM}$ paclitaxel. Each dot represents a replicate well. Horizontal lines signify mean and SD. (F) Graph of relative cell numbers following transfection of siCON or siRNAs targeting regulators of autophagy. Data were normalized to cell number in siCON/vehicle control and siCON/paclitaxel for paclitaxel-treated experiments. Error bars represent SDs across six replicates/condition. (G) Graph of relative cell number in paclitaxel-treated compared to vehicle control following transfection of siCON, siCERT, siATG7 or siCERT and siATG7. Treated/control ratio for siCON was set to 1. Error bars represent SDs across six replicates/condition. 
autophagosomal structures in siCERT/paclitaxeltreated cells in contrast to siCON/paclitaxel-treated cells (see Supporting information, Figures S2A, B. Percentage of cells with electron-dense structures with siCERT/paclitaxel: $75 \%, n=24$ versus siCON/ paclitaxel: $44 \%, n=27, p=0.045$, Fisher's exact test). In contrast, co-depletion of CERT and LAMP2 in the presence of paclitaxel significantly reduced the number of cells with these electron-dense structures compared to siCERT/paclitaxel-treated cells (siCERT/ siLAMP2/paclitaxel: $32 \%$ versus siCERT/Paclitaxel $75 \%, n=25$ and $\mathrm{n}=24$ respectively; $p=0.004$, Fisher's exact test), suggesting that specific changes to the cellular ultrastructure occur in siCERT/paclitaxeltreated cells (see Supporting information, Figure S2A).

\section{Relationship of paclitaxel response with autophagy gene expression}

To explore the relationship of autophagy with paclitaxel response, we addressed whether silencing other components of the autophagy pathway might influence paclitaxel sensitivity. Silencing ATG7 induced paclitaxel resistance, indicating that a proximal member of the autophagy pathway may influence paclitaxel response (Figure 2F). Phenocopying LAMP2 silencing, co-depletion of both ATG7 and CERT significantly attenuated CERT-mediated cell death (Mann-Whitney U-test, $p=0.002$ ) (Figure 2G).

\section{CERT depletion promotes mitotic catastrophe that is LAMP2-dependent}

We sought to establish the cell-cycle fate of individual cells following paclitaxel treatment and CERT silencing, through live cell microscopy imaging of H2B-GFP-labelled HCT116 cells during $48 \mathrm{~h}$ of paclitaxel exposure. Paclitaxel stabilizes microtubules, resulting in mitotic arrest [16] leading to death in mitosis or death following mitotic slippage into interphase [17]. Paclitaxel treatment of siCON-transfected cells promoted cell death following mitosis, or in interphase cells following exposure to anti-mitotic agents as previously reported [18]. CERT depletion induced two-fold more death events over $48 \mathrm{~h}$ compared to control transfected cells following paclitaxel treatment (Figure 3A, B) that mostly occurred during a multinucleated interphase resulting from an aberrant mitosis. Co-depletion of LAMP2 with CERT reversed paclitaxel sensitization during $48 \mathrm{~h}$ of paclitaxel exposure (Figure 1C). Paclitaxel-induced cell death was still observed in interphase cells but LAMP2 depletion abrogated the majority of mitotic and post-mitotic cell death events, with the majority of multinucleated cells surviving to the end of the observation period. Taken together, these data suggest that CERT depletion promotes the elimination of multinucleated polyploid cells following paclitaxel exposure and this is LAMP2-dependent.
CERT is over-expressed in HER2 ${ }^{+}$primary breast cancer

Next, we determined the expression of CERT in a panel of cancer cell lines by qPCR to attempt to identify cancer subtypes in which CERT expression may play a role (Figure 4A). Breast cancer cell lines displayed the broadest distribution of CERT expression. Analysis of four HER2 ${ }^{+}$and eight HER2 ${ }^{-}$breast cancer cell lines revealed higher relative CERT expression in the HER2 ${ }^{+}$cell lines compared to HER2 ${ }^{-}$cancer cell lines ( $p=0.006, t$-test; Figure 4A). We identified 12 breast cancer datasets for which CERT expression was measured by microarray analysis. CERT mRNA expression was significantly repressed in basal breast cancers ( $p=1.41 \mathrm{e}-51)$ relative to other intrinsic subtypes of breast cancer (see Supporting information, Figure S3A, B). Next we assessed CERT protein expression in a tissue microarray (TMA) panel of 356 primary breast cancers of mixed ER/HER2 receptor subtype, tumour grade and nodal status (Table 1). CERT protein expression, determined by the quickscore (CERT expression intensity $\times$ percentage of cells positive) [7], was significantly elevated in HER2 ${ }^{+}$relative to HER $2^{-}$primary breast cancers (Wilcoxon test, $p=0.0009$; Figure 4B; for representative images; see Supporting information, Figure S4A) with no significant difference between $\mathrm{ER}^{+}$and $\mathrm{ER}^{-}$subtypes $(p=0.0889)$.

CERT depletion sensitizes HER2 ${ }^{+}$breast cancer cell lines to cytotoxics and induces autophagic flux

Next we assessed drug sensitivity in three HER2 ${ }^{+}$ breast cancer cell lines, BT474, HCC1954 and SKBR3, following CERT silencing. CERT depletion resulted in multidrug sensitization to clinically relevant cytotoxics, paclitaxel, doxorubicin and cisplatin (Figure 4C). CERT depletion in the HCC1954 cell line resulted in decreased levels of LC3-I and LC3-II following treatment with paclitaxel, cisplatin or doxorubicin when compared to siCON-transfected cells (Figure 4D), suggesting that, concordant with the HCT116 analyses, increased autophagic flux occurs following CERT depletion and cytotoxic drug treatment in HER2 ${ }^{+}$ breast cancer cells.

Next, the HCC1954 cell line was transfected with siCON, siCERT, SILAMP2 or siCERT and siLAMP2 and treated with either paclitaxel or doxorubicin, used in the management of high-risk breast cancer in the adjuvant setting (Figure 4E). In all conditions, LAMP2 co-silencing resulted in a significant attenuation of CERT depletion-induced drug sensitization (Mann-Whitney U-test: paclitaxel $50 \mathrm{nM}$, $p=0.0087$; paclitaxel $500 \mathrm{nM}, p=0.0043$; doxorubicin $250 \mathrm{nM}, p=0.0087$; doxorubicin $1000 \mathrm{nM}, p=$ 0.015). CERT depletion also resulted in trastuzumab sensitization (see Supporting information, Figure S4B; Mann-Whitney U-test, $p=0.0087$ ). 

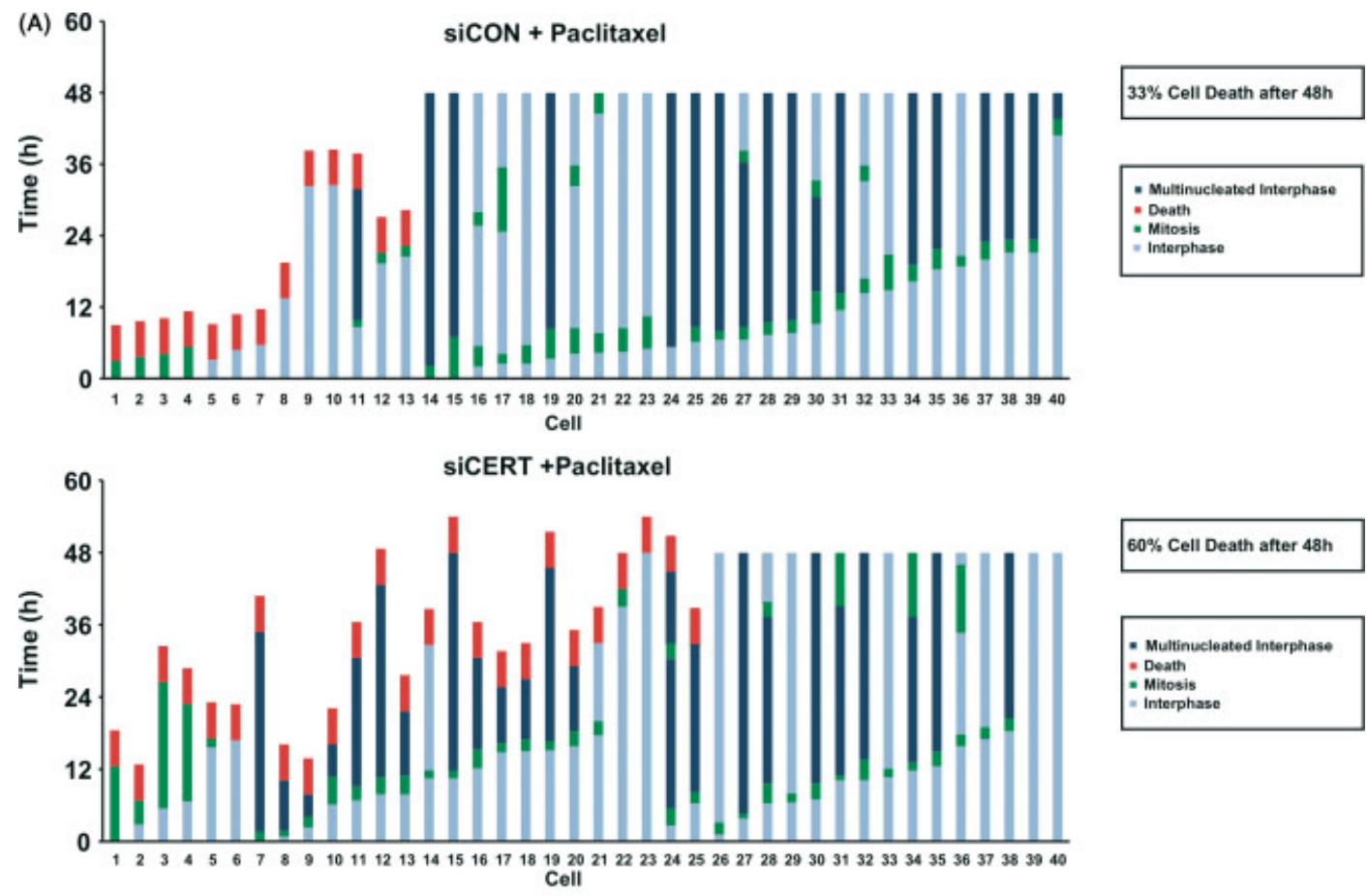

$60 \%$ Cell Death after $48 \mathrm{~h}$
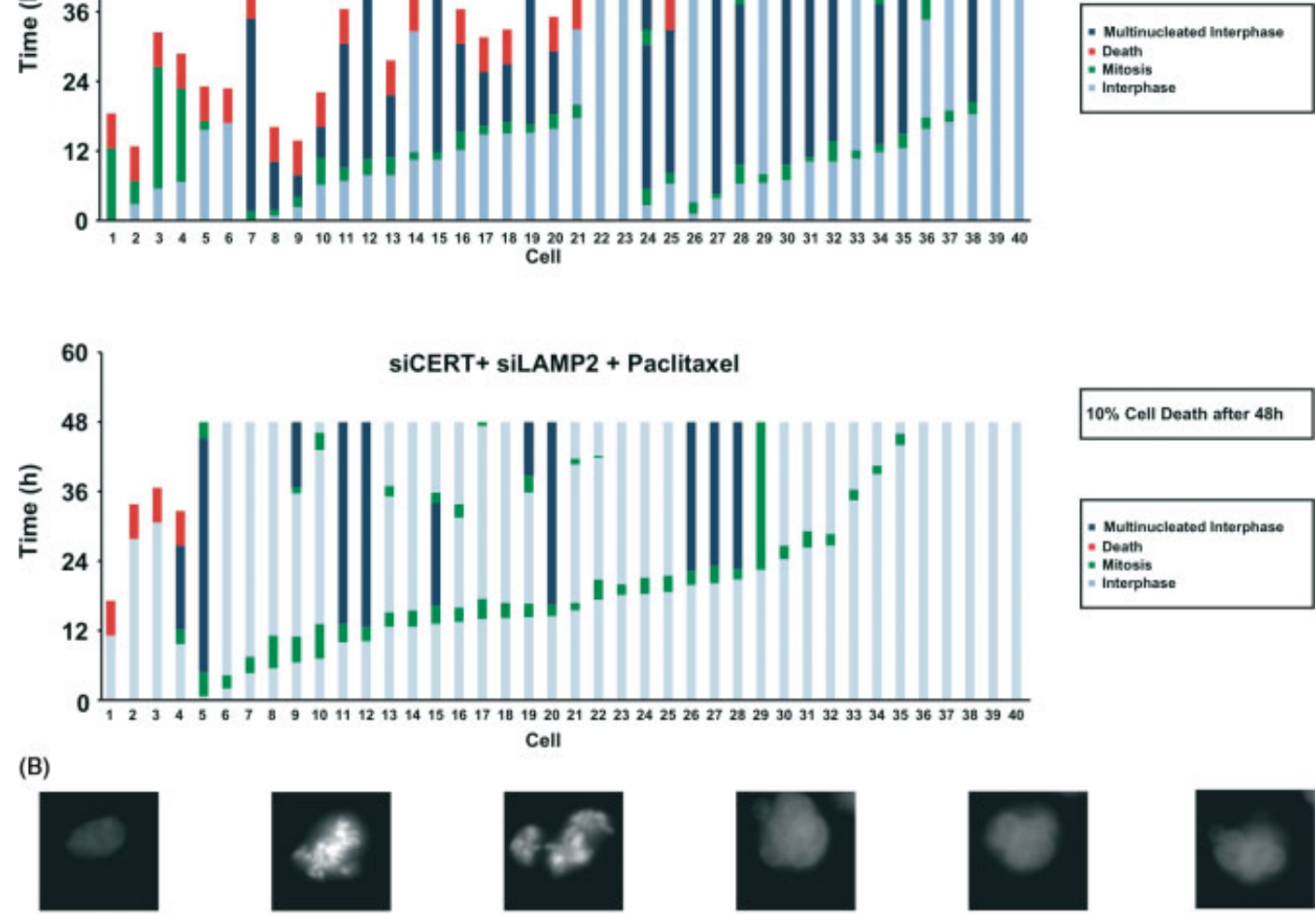

siCERT + siLAMP2 + Paclitaxel

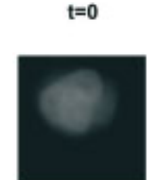

$\mathrm{t}=720$

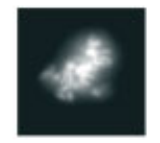

$\mathrm{t}=120$

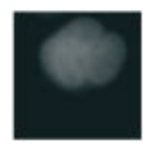

$t=840$

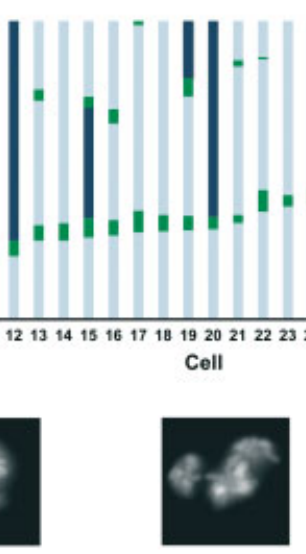

$\mathrm{t}=240$

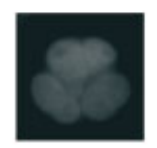

$t=960$

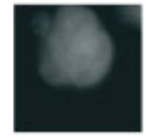

$\mathrm{t}=360$

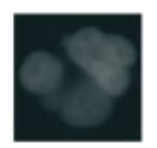

$\mathrm{t}=1080$
$10 \%$ Cell Death after $48 \mathrm{~h}$

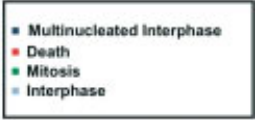

Figure 3. Live cell imaging and single cell fate analysis following CERT silencing and paclitaxel treatment. (A) Graph of single cell fate analysis during paclitaxel exposure following transfection with siCON, siCERT, or siCERT and siLAMP2. Cells were individually tracked for $48 \mathrm{~h}$ following paclitaxel treatment. Timing of entry into mitosis was scored from the time of nuclear envelope breakdown (NEB) to reformation of nuclei. (B) Example of a time-lapse sequence ( $t$, time in $\min$ ) of a CERT-depleted cell treated with paclitaxel over $1200 \mathrm{~min}$ following mitotic entry. The cell undergoes nuclear envelope breakdown (NEB), followed by an aberrant mitosis resulting in a multinucleated interphase and subsequently cell death.

CERT expression is associated with breast cancer adjuvant chemotherapy survival outcome

Next, we attempted to address whether low CERT expression might be associated with improved breast cancer-specific survival (BCSS), particularly in primary breast cancers treated with adjuvant chemotherapy in the 356 primary breast cancer TMA cohort. We used maximally selected rank statistics [8] to derive a threshold for separating tumours into cohorts of low and high CERT expression based on survival outcome. This method selects an optimal threshold for prediction, avoiding the erroneous reporting of over-optimistic low $p$ values by adjusting for multiple testing inherent in the selection of the threshold. We obtained a CERT 
(A)

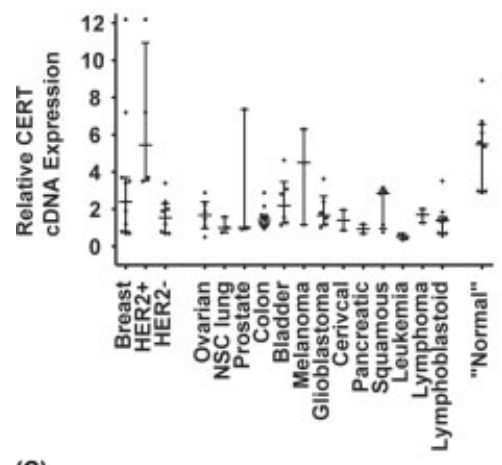

(C)
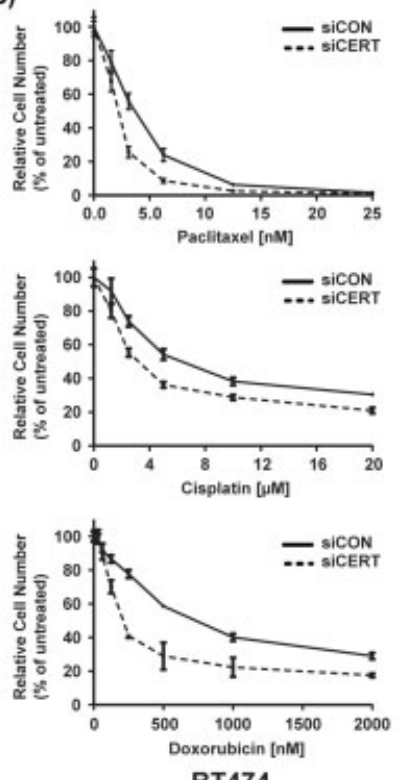

BT474

(D)
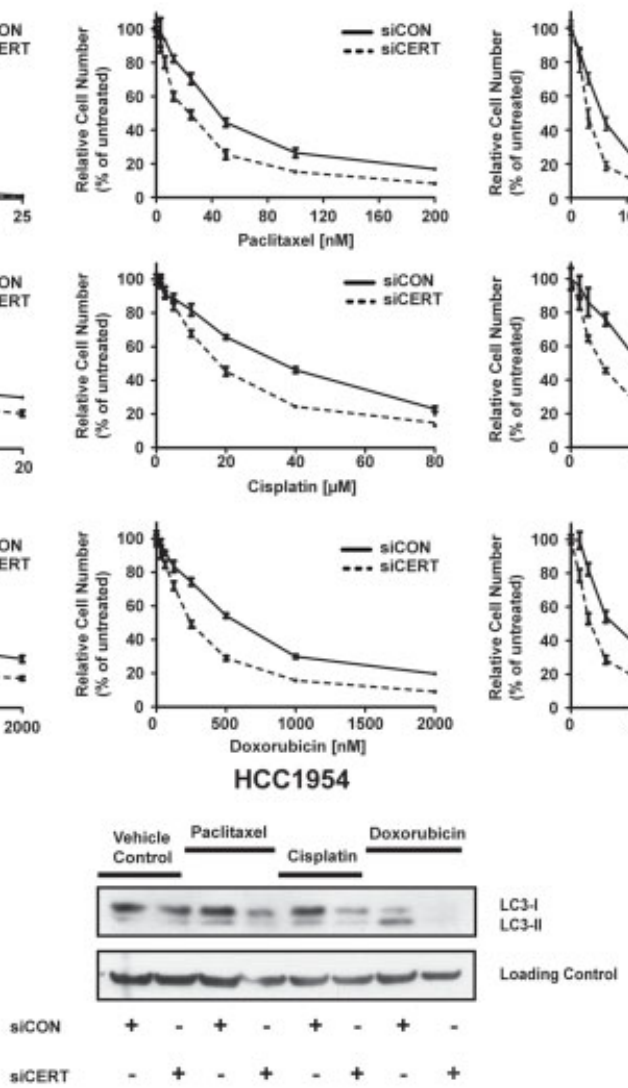

(B)
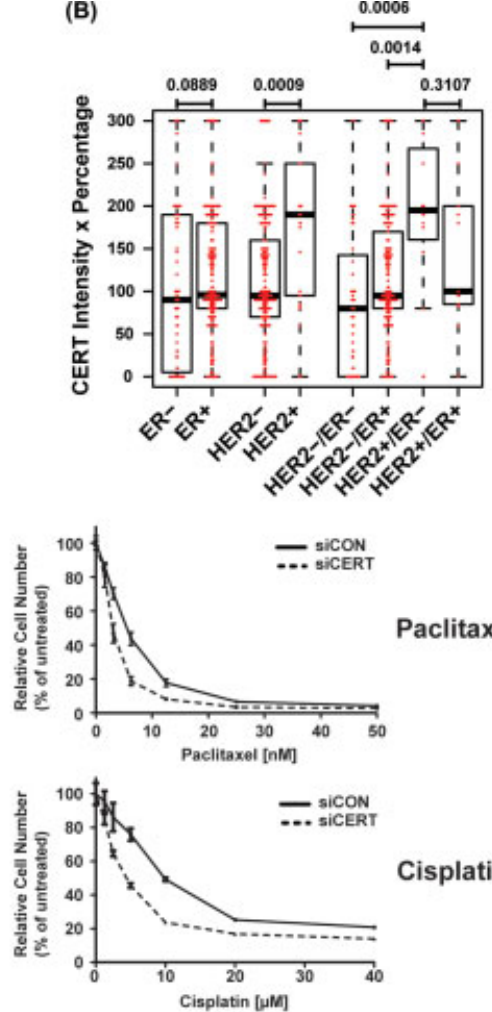

Cisplatin

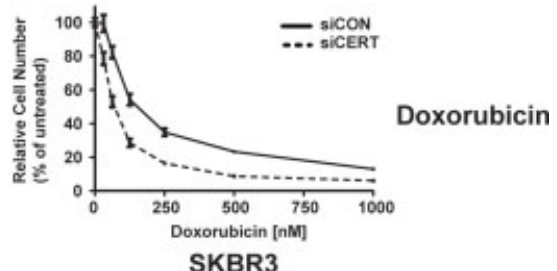

SKBR3
(E)

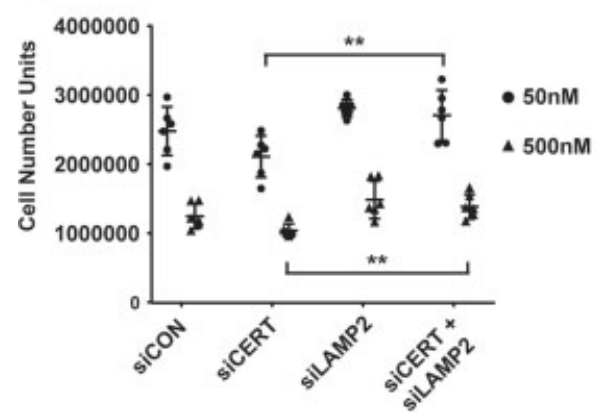

Doxorubicin

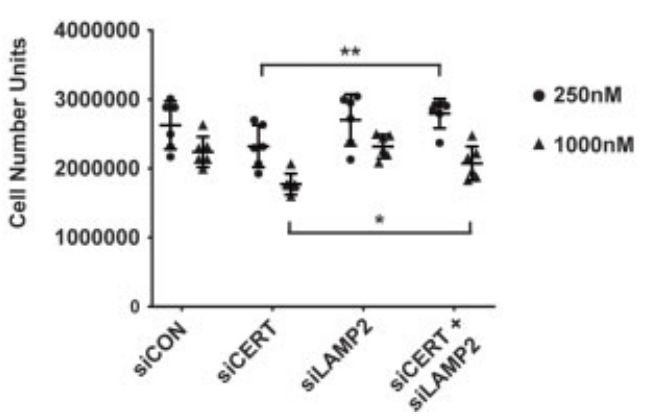

Figure 4. CERT is over-expressed in HER2 ${ }^{+}$primary breast cancer and is associated with poor prognosis. (A) Graph of relative CERT cDNA expression in cell lines of various tissue origins. Horizontal lines signify median and interquartile range. (B) Graph demonstrates CERT protein intensity in a breast cancer TMA cohort of 356 primary breast cancers divided into various subtypes, based on ER and HER2 receptor status. The length of the whiskers was limited by maximal $=1.5$ times the interquartile range. (C) Graph showing relative cell number compared to vehicle control of HER2 ${ }^{+}$breast cancer cell lines, BT474, HCC1954 and SKBR3, following either siCON or siCERT transfection and treatment with paclitaxel, cisplatin or doxorubicin. (D) Western blot demonstrating LC3-I and LC3-II expression in HCC1954 cells following $48 \mathrm{~h}$ of CERT depletion, followed by $24 \mathrm{~h}$ of treatment with paclitaxel, cisplatin or doxorubicin. (E) Scatter plot of cell number (as measured by fluorescence activity) in paclitaxel-treated $(50$ and $500 \mathrm{nM})$ (left) and doxorubicin-treated (250 and $1000 \mathrm{nM})$ (right) HCC1954 cells following transfection with siCON, siCERT, siLAMP2 or siCERT and siLAMP2. Each dot represents a replicate well. Horizontal lines signify mean and SD. 
Table 1. Demographic data of patients in breast cancer TMA cohort

\begin{tabular}{|c|c|c|c|}
\hline & All patients $(n=426)$ & CERT low $(n=271)$ & CERT high $(n=102)$ \\
\hline Median age, years (range) & $58(27-92)$ & $58(30-90)$ & $59(27-92)$ \\
\hline Unknown & 2 & 2 & 0 \\
\hline Median size, mm (range) & $20(1-130)$ & $19(1-100)$ & $20(6-130)$ \\
\hline Unknown & 13 & 11 & 1 \\
\hline \multicolumn{4}{|l|}{ Grade, $n(\%)$} \\
\hline 1 & $98(23)$ & $67(25)$ & $11(11)$ \\
\hline 2 & $184(43)$ & $127(47)$ & $43(42)$ \\
\hline 3 & $143(34)$ & $76(28)$ & $48(47)$ \\
\hline Unknown & 1 & 1 & 0 \\
\hline \multicolumn{4}{|l|}{ Lymph node status, $n(\%)$} \\
\hline Positive & $215(53)$ & $125(48)$ & $63(65)$ \\
\hline Negative & $192(47)$ & $134(52)$ & 34 (35) \\
\hline Unknown & 19 & 12 & 5 \\
\hline \multicolumn{4}{|l|}{ Stage, $n(\%)$} \\
\hline $\mathrm{T} 1,0-2 \mathrm{~cm}$ & $246(60)$ & $163(62)$ & $54(53)$ \\
\hline $\mathrm{T} 2,2-5 \mathrm{~cm}$ & $149(36)$ & $90(35)$ & 39 (39) \\
\hline $\mathrm{T} 3>5 \mathrm{~cm}$ & $18(4)$ & $7(3)$ & $8(8)$ \\
\hline Unknown & 13 & 11 & 1 \\
\hline \multicolumn{4}{|l|}{ ER status, $n(\%)$} \\
\hline Positive & $326(78)$ & $216(80)$ & $75(74)$ \\
\hline Negative & $92(22)$ & $54(20)$ & $27(26)$ \\
\hline Unknown & 8 & 1 & 0 \\
\hline \multicolumn{4}{|l|}{ HER2 status, $n(\%)$} \\
\hline Positive & $31(7)$ & $11(4)$ & $18(18)$ \\
\hline Negative & $393(93)$ & $258(96)$ & $84(82)$ \\
\hline Unknown & 2 & 2 & 0 \\
\hline \multicolumn{4}{|l|}{ PR status, $n(\%)$} \\
\hline Positive & $300(79)$ & 198 (78) & 79 (79) \\
\hline Negative & $80(21)$ & $57(22)$ & $21(21)$ \\
\hline Unknown & 46 & 16 & 2 \\
\hline \multicolumn{4}{|l|}{ Chemotherapy, $n(\%)$} \\
\hline Yes & $149(49)$ & $100(48)$ & $41(49)$ \\
\hline No & $157(51)$ & $107(52)$ & $43(51)$ \\
\hline Unknown & 120 & 64 & 18 \\
\hline
\end{tabular}

quickscore of $<175$ and $>175$ for separating tumours into low and high CERT expression, respectively. Significantly poorer breast cancer outcome was observed across all patients with primary breast cancers, with a CERT quickscore $>175$ compared to patients with a CERT quickscore $<175$ (HR 2.295\% CI 1.3-3.6, $p=$ 0.003; Figure 5A). A similar relationship was observed when the analysis was restricted to HER2 ${ }^{+}$breast cancer patients only (Figure 5B; $p=0.035 \mathrm{HR}$ 6.81). In a multivariate Cox regression model, the association of CERT expression with survival acts as a significant independent prognostic variable (Table 2). The relationship between CERT expression and relapse-free survival was further supported in a meta-analysis of 1861 patients with primary breast cancer, using the publicly available Kaplan-Meier meta-analysis tool [19] (see Supporting information, Figure S4C), HR 1.2 (1.1-1.4), $p=0.027)$.

We asked whether the relationship of low CERT expression with significantly improved outcome was observed when patients were stratified by use of adjuvant chemotherapy. Low CERT expression in the 356 patient TMA cohort was associated with significantly improved outcome in patients treated with adjuvant chemotherapy [Figure 5C, $n=134$, HR 2.2 (1.2-5.5), $p=0.014]$. In a similarly powered analysis of 150 patients who did not receive adjuvant chemotherapy from within this cohort, there was no such significant association of low CERT expression with improved outcome (Figure 5D), suggesting that CERT expression may predict outcome, specifically in patients with primary breast cancer receiving chemotherapy.

\section{Discussion}

Through transcriptomic and functional genomics-based approaches, we have identified that CERT depletion and paclitaxel exposure accelerate autophagic flux in both colon and breast cancer models and results in LAMP2-dependent death of multinucleated cells. Accumulating evidence supports the importance of autophagy in the response to cytotoxic drug or radiation therapy that may enhance death or augment cancer cell viability [20-24]. We previously identified the lysosomal enzymes GBA1 and GBA3 as regulators of paclitaxel sensitivity [2], and microtubule stabilizing drugs initiate lysosomal cathepsin B activation and release, inhibition of which promotes resistance to this class of drug [14].

Expression of an autophagy gene module is significantly enriched following CERT silencing and 

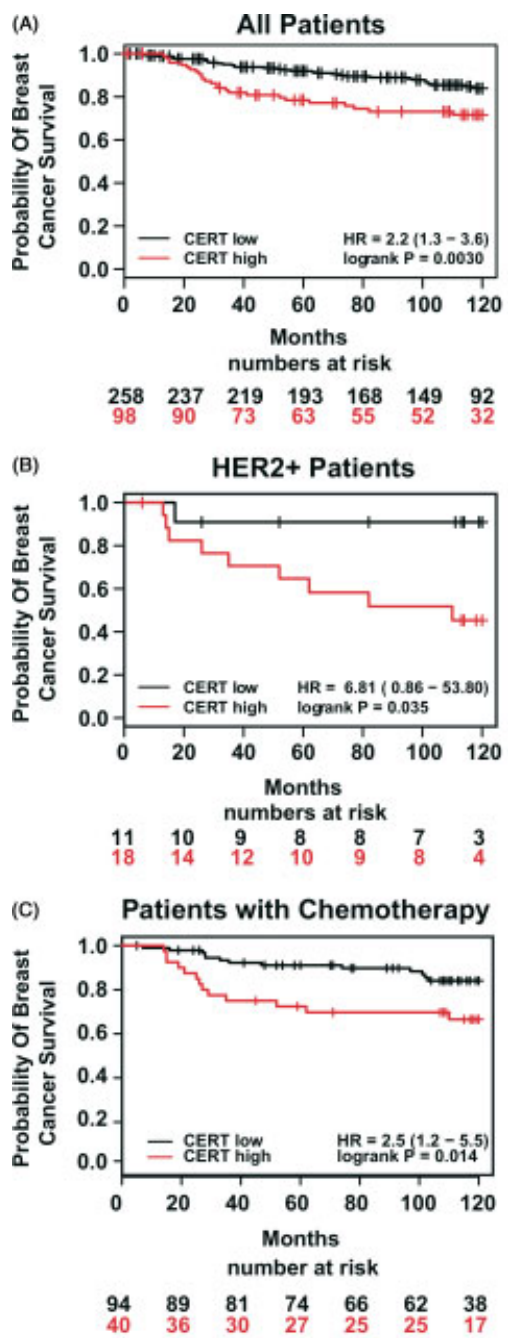

(D) Patients without Chemotherapy

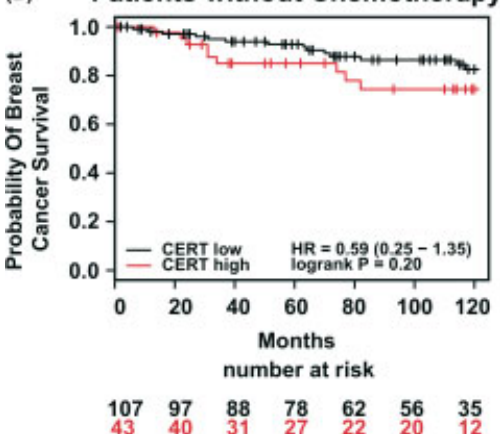

Figure 5. CERT expression is associated with survival outcome [breast cancer-specific survival (BCSS)] in patients with primary breast cancer treated with adjuvant chemotherapy. (A) CERT immunohistochemistry in 356 primary breast cancers and Kaplan-Meier analysis of high CERT expression compared to tumours with low CERT expression (quickscore $>175$ versus $<175$ ). (B) Kaplan-Meier analysis of high CERT expression compared to tumours with low CERT expression (quickscore $>175$ versus $<175$ ), restricted to HER2 ${ }^{+}$breast cancer patients $(n=29)$ within the same cohort of 356 patients. (C) Kaplan-Meier analysis of high CERT expression compared to tumours with low CERT expression (quickscore $>175$ versus $<175$ ), restricted to patients treated with adjuvant chemotheraphy $(n=134)$ within the same cohort of 356 patients. (D) Kaplan-Meier analysis of high CERT expression compared to tumours with low CERT expression (quickscore $>175$ versus $<175)$, restricted to patients treated without adjuvant chemotheraphy $(n=150)$ within the same cohort of 356 patients. ceramide treatment. We suggest that the autophagy transcriptional response may reflect accumulation of ceramide within the ER, consistent with previous reports of autophagy initiation by ceramide $[25,26]$ and the transcriptional regulation of autophagy [27]. Through this analysis, we suggest an explanation for the synergistic activities of CERT depletion and paclitaxel exposure that may provide a molecular basis for the elimination of multinucleated polyploid cells following an aberrant mitosis. We speculate that expression of LAMP2 primes the cell for completion of the autophagic process, but alone is insufficient to drive cell death. Cell death following CERT depletion and drug exposure may result from the combination of the synergistic rise in ER-restricted cellular ceramide and autophagy gene expression coordinated with LAMP2 expression. Consistent with this model, we observed co-localization of LAMP2 and LC3 in the presence of lysosomal inhibitors in HEK293 cells, as well as a reduction of LC3-I and LC3-II in CERT-depleted cells treated with paclitaxel. Furthermore, abrogation of the siCERT multidrug sensitizing phenotype and the death of multinucleated cells was observed following LAMP2 silencing.

Multinucleation may be a precursor of mononucleate aneuploidy/polyploidy and CIN [28-30]. We have shown that autophagic flux and the consequent death of multinucleated cells following paclitaxel exposure is dependent upon LAMP2 induction following CERT depletion. Aneuploid cells display an increased susceptibility to compounds that interfere with protein folding, and trisomic MEFs display increased LC3 lipidation relative to wild-type MEFs [31]. These data support our observations that CERT depletion and paclitaxel treatment induces the unfolded protein response [2], autophagic flux and the specific death of multinucleated cells.

CERT is relatively over-expressed in HER $2^{+}$breast cancer and CERT silencing sensitizes HER2 ${ }^{+}$breast cancer cell lines to paclitaxel, doxorubicin, cisplatin and trastuzumab. Cytotoxic multidrug sensitization is associated with accelerated autophagic flux, which appears to be LAMP2-dependent. Consistent with the drug-sensitizing phenotype following CERT silencing, low expression of CERT predicts improved breast cancer outcome following adjuvant chemotherapy. Prospective validation of the potential role for CERT in predicting outcome following adjuvant chemotherapy in breast cancer is in progress.

In summary, CERT expression may confer prognostic utility in breast cancer and there may be therapeutic potential in the targeting of CERT to augment autophagy and the death of multinucleated cells to limit the initiation of chromosomal instability, intra-tumour heterogeneity and chemotherapy resistance.

\section{Acknowledgment}

We thank the following: Sarah McClelland, Jasmin Zohren and Rebecca Burrell for help with manuscript 
Table 2. Multivariate analysis of prognostic predictors including CERT expression in breast cancer TMA cohort

$\begin{array}{lcccc} & \log (\mathrm{HR}) & \text { HR } & \text { se[log(HR)] } & p \\ \text { Size, } \mathrm{mm} & 0.02005 & 1.020 & 0.0084 & 0.017 \\ \text { Grade 2 } & 1.66957 & 5.310 & 1.0356 & 0.110 \\ \text { Grade 3 } & 2.87047 & 17.65 & 1.0224 & 0.005 \\ \text { Node-positive } & 0.49507 & 1.64 & 0.3477 & 0.150 \\ \text { CERT } & 0.00365 & 1.004 & 0.0017 & 0.030\end{array}$

preparation; Hannah Armer and Lucy Collinson from the EM facility at Cancer Research UK (CR-UK) LRI and Niamh Murphy for help with the breast cancer TMA cohort. CS is funded by the Medical Research Council and CR-UK. AL, NM, AR, JD and ST are funded by $\mathrm{CR}-\mathrm{UK}$. $\mathrm{ZS}$ is funded by the National Institute of Health (Grant Nos NCI SPORE P50 CA 89393 and R21LM008823-01A1), the Danish Council for Independent Research/Medical Sciences (FSS) and the Breast Cancer Research Foundation (BCRF).

\section{Author contributions}

AL performed and analysed most of the experiments in this study and participated in their design and the writing of this manuscript. RR and PG analysed the breast cancer TMA data provided by VS and AH. JS, DE and MK performed statistical analyses. NJ performed CERT expression studies in cell lines and drug response assays in HER $2^{+}$cell lines. PE analysed CERT exon array data. BN prepared samples for the CERT exon array. SS and LO performed mass spectrometry for ceramide content. NB and ZS performed survival analysis in breast cancer datasets. AR helped with validation of siRNA transfection. NM helped with initial autophagy experiments. JD and ST provided critical suggestions and discussions. CS conceived and supervised this study and the writing of the manuscript.

\section{References}

1. Ogretmen B, Hannun YA. Biologically active sphingolipids in cancer pathogenesis and treatment. Nat Rev Cancer 2004; 4: 604-616.

2. Swanton C, Marani M, Pardo O, et al. Regulators of mitotic arrest and ceramide metabolism are determinants of sensitivity to paclitaxel and other chemotherapeutic drugs. Cancer Cell 2007; 11: $498-512$.

3. Hanada K, Kumagai K, Yasuda S, et al. Molecular machinery for non-vesicular trafficking of ceramide. Nature 2003; 426: 803-809.

4. Juul N, Szallasi Z, Eklund AC, et al. Assessment of an RNA interference screen-derived mitotic and ceramide pathway metagene as a predictor of response to neoadjuvant paclitaxel for primary triplenegative breast cancer: a retrospective analysis of five clinical trials. Lancet Oncol 2010; 11: 358-365.

5. Swanton C, Nicke B, Schuett M, et al. Chromosomal instability determines taxane response. Proc Natl Acad Sci USA 2009; 106: 8671-8676.

6. Lee AJX, Endesfelder D, Rowan AJ, et al. Chromosomal instability confers intrinsic multidrug resistance. Cancer Res 2011; 71: $1858-1870$.
7. Detre S, Saclani Jotti G, Dowsett M. A 'quickscore' method for immunohistochemical semiquantitation: validation for oestrogen receptor in breast carcinomas. J Clin Pathol 1995; 48: 876-878.

8. Hothorn T, Lausen B. On the exact distribution of maximally selected rank statistics. Comput Stat Data Anal 2003; 43: 121-137.

9. Hothorn T, Lausen B. Maximally selected rank statistics in R. $R$ News 2002; 2: 3-5.

10. Toth B, Balla A, Ma H, et al. Phosphatidylinositol 4-kinase III $\beta$ regulates the transport of ceramide between the endoplasmic reticulum and Golgi. J Biol Chem 2006; 281: 36369-36377.

11. Lee AJ, East P, Pepper S, et al. Concordance of exon array and real-time PCR assessment of gene expression following cancer cell cytotoxic drug exposure. Cell Cycle 2008; 7: 3947-3948.

12. Saftig P, Beertsen W, Eskelinen EL. LAMP-2: a control step for phagosome and autophagosome maturation. Autophagy 2008; 4: 510-512.

13. Mehrpour M, Esclatine A, Beau I, et al. Overview of macroautophagy regulation in mammalian cells. Cell Res 2010; 20: $748-762$.

14. Broker LE, Huisman C, Span SW, et al. Cathepsin B mediates caspase-independent cell death induced by microtubule stabilizing agents in non-small cell lung cancer cells. Cancer Res 2004; 64: $27-30$.

15. Groth-Pedersen L, Ostenfeld MS, Hoyer-Hansen M, et al. Vincristine induces dramatic lysosomal changes and sensitizes cancer cells to lysosome-destabilizing siramesine. Cancer Res 2007; 67: 2217-2225.

16. Schiff PB, Horwitz SB. Taxol stabilizes microtubules in mouse fibroblast cells. Proc Natl Acad Sci USA 1980; 77: 1561-1565.

17. Blagosklonny MV. Mitotic arrest and cell fate: why and how mitotic inhibition of transcription drives mutually exclusive events. Cell Cycle 2007; 6: 70-74.

18. Gascoigne KE, Taylor SS. Cancer cells display profound intraand interline variation following prolonged exposure to antimitotic drugs. Cancer Cell 2008; 14: 111-122.

19. Gyorffy B, Lanczky A, Eklund AC, et al. An online survival analysis tool to rapidly assess the effect of 22277 genes on breast cancer prognosis using microarray data of 1809 patients. Breast Cancer Res Treat 2010; 123: 725-731.

20. de Bruin EC, Medema JP. Apoptosis and non-apoptotic deaths in cancer development and treatment response. Cancer Treat Rev 2008; 34: 737-749.

21. Demasters G, Di X, Newsham I, et al. Potentiation of radiation sensitivity in breast tumor cells by the vitamin D3 analogue, EB 1089, through promotion of autophagy and interference with proliferative recovery. Mol Cancer Ther 2006; 5: 2786-2797.

22. Gewirtz DA, Hilliker ML, Wilson EN. Promotion of autophagy as a mechanism for radiation sensitization of breast tumor cells. Radiother Oncol 2009; 92: 323-328.

23. Hoyer-Hansen M, Jaattela M. Autophagy: an emerging target for cancer therapy. Autophagy 2008; 4: 574-580.

24. Karna P, Zughaier S, Pannu V, et al. Induction of reactive oxygen species-mediated autophagy by a novel microtubule-modulating agent. J Biol Chem 2010; 285: 18737-18748. 
25. Daido S, Kanzawa T, Yamamoto A, et al. Pivotal role of the cell death factor BNIP3 in ceramide-induced autophagic cell death in malignant glioma cells. Cancer Res 2004; 64: 4286-4293.

26. Spassieva SD, Mullen TD, Townsend DM, et al. Disruption of ceramide synthesis by CerS2 down-regulation leads to autophagy and the unfolded protein response. Biochem J 2009; 424: 273-283.

27. Settembre C, Di Malta C, Polito VA, et al. TFEB links autophagy to lysosomal biogenesis. Science 2011; 332: 1429-1433.

28. Tatsuka M, Katayama H, Ota T, et al. Multinuclearity and increased ploidy caused by overexpression of the Aurora- and Ipl1like midbody-associated protein mitotic kinase in human cancer cells. Cancer Res 1998; 58: 4811-4816.

29. Fujiwara T, Bandi M, Nitta M, et al. Cytokinesis failure generating tetraploids promotes tumorigenesis in p53-null cells. Nature 2005; 437: 1043-1047.

30. Shi Q, King RW. Chromosome nondisjunction yields tetraploid rather than aneuploid cells in human cell lines. Nature 2005; 437: $1038-1042$

31. Tang Y-C, Williams BR, Siegel JJ, et al. Identification of aneuploidy-selective antiproliferation compounds. Cell 2011; 144: 499-512.

32. *Irizarry RA, Hobbs B, Collin F, et al. Exploration, normalization, and summaries of high density oligonucleotide array probe level data. Biostatistics 2003; 4: 249-264.

33. *Gentleman RC, Carey VJ, Bates DM, et al. Bioconductor: open software development for computational biology and bioinformatics. Genome Biol 2004; 5: R80.

34. *Subramanian A, Tamayo P, Mootha VK, et al. Gene set enrichment analysis: a knowledge-based approach for interpreting genome-wide expression profiles. Proc Natl Acad Sci USA 2005; 102: $15545-15550$.

35. *Mootha VK, Lindgren CM, Eriksson KF, et al. PGC-1 $\alpha$ responsive genes involved in oxidative phosphorylation are coordinately downregulated in human diabetes. Nat Genet 2003; 34: 267-273.

36. *Bielawski J, Pierce JS, Snider J, et al. Comprehensive quantitative analysis of bioactive sphingolipids by high-performance liquid chromatography-tandem mass spectrometry. Methods Mol Biol 2009; 579: 443-467.

37. *Van Veldhoven PP, Bell RM. Effect of harvesting methods, growth conditions and growth phase on diacylglycerol levels in cultured human adherent cells. Biochim Biophys Acta 1988; 959: 185-196.
38. *Chin K, DeVries S, Fridlyand J, et al. Genomic and transcriptional aberrations linked to breast cancer pathophysiologies. Cancer Cell 2006; 10: 529-541.

39. *Desmedt C, Haibe-Kains B, Wirapati P, et al. Biological processes associated with breast cancer clinical outcome depend on the molecular subtypes. Clin Cancer Res 2008; 14: 5158-5165.

40. *Minn AJ, Gupta GP, Siegel PM, et al. Genes that mediate breast cancer metastasis to lung. Nature 2005; 436: 518-524.

41. *Pawitan Y, Bjohle J, Amler L, et al. Gene expression profiling spares early breast cancer patients from adjuvant therapy: derived and validated in two population-based cohorts. Breast Cancer Res 2005; 7: R953-964.

42. * Sotiriou C, Wirapati P, Loi S, et al. Gene expression profiling in breast cancer: understanding the molecular basis of histologic grade to improve prognosis. J Natl Cancer Inst 2006; 98: 262-272.

43. *Wang Y, Klijn JG, Zhang Y, et al. Gene-expression profiles to predict distant metastasis of lymph-node-negative primary breast cancer. Lancet 2005; 365: 671-679.

44. *Andre F, Job B, Dessen P, et al. Molecular characterization of breast cancer with high-resolution oligonucleotide comparative genomic hybridization array. Clin Cancer Res 2009; 15: 441-451.

45. *Hess KR, Anderson K, Symmans WF, et al. Pharmacogenomic predictor of sensitivity to preoperative chemotherapy with paclitaxel and fluorouracil, doxorubicin, and cyclophosphamide in breast cancer. J Clin Oncol 2006; 24: 4236-4244.

46. *Miller LD, Smeds J, George J, et al. An expression signature for p53 status in human breast cancer predicts mutation status, transcriptional effects, and patient survival. Proc Natl Acad Sci USA 2005; 102: 13550-13555.

47. ${ }^{*}$ Lu X, Wang ZC, Iglehart JD, et al. Predicting features of breast cancer with gene expression patterns. Breast Cancer Res Treat 2008; 108: 191-201.

48. ${ }^{*} \mathrm{Li}$ Y, Zou L, Li Q, et al. Amplification of LAPTM4B and YWHAZ contributes to chemotherapy resistance and recurrence of breast cancer. Nat Med 2010; 16: 214-218.

49. ${ }^{*}$ http://www.ncbi.nlm.nih.gov/projects/geo/query/acc.cgi?acc= GSE2109

* These references were cited in the Supporting information only.

\section{SUPPORTING INFORMATION ON THE INTERNET}

The following supporting information may be found in the online version of this article:

Supplementary materials and methods

Figure S1. Validation of siRNA transfection, measurements of ceramide content of cells, detection of LC3-I and LC3-II by western blot after the addition of leupeptin protease inhibitor and corrected caspase 3/7 activity following co-silencing of LAMP2

Figure S2. Representative transmission electron microscopy images of siCON/paclitaxel and siCERT/paclitaxel cells

Figure S3. Analysis of CERT expression across 12 microarray datasets of breast cancer, divided into subtypes

Figure S4. Representative CERT immunohistochemistry images, CERT sensitization data for trastuzumab and Kaplan-Meier relapse-free survival curves for patients with tumours with high CERT expression compared to those with low CERT expression 